\title{
General method for the quantification of drug loading and release kinetics of nanocarriers
}

\author{
Richard Schwarzl ${ }^{\mathrm{a}, *}$, Fang Du ${ }^{\mathrm{b}}$, Rainer Haag ${ }^{\mathrm{b}}$, Roland R. Netz \\ ${ }^{a}$ Freie Universität Berlin, Fachbereich Physik, Berlin, Germany \\ ${ }^{b}$ Freie Universität Berlin, Institut für Chemie und Biochemie, Berlin, Germany
}

\begin{abstract}
Macromolecular nanostructures that are used as drug carriers are characterized by their loading and release kinetics. Release studies commonly employ the dialysis method, in which a cellulose membrane separates the solution of released drug from the nanocarrier solution. We demonstrate that it is necessary to take the effect of the dialysis membrane on the release kinetics into account. Using a two-step approach, consisting of a calibration experiment of drug diffusion through the dialysis membrane in the absence of nanocarriers, and an experiment in the presence of nanocarriers, we are able to determine all kinetic rates and in particular to disentangle kinetic dialysis membrane properties from kinetic nanocarrier properties. We apply our general approach to experimental dexamethasone release data from core-multishell nanocarriers and demonstrate that our method yields a consistent description of the nanocarrier release kinetics.

Keywords: dexamethasone, core-multishell (CMS) nanocarriers, drug delivery systems, dialysis, drug release, rate equations, partition coefficients, retention, release and loading rates, rate disentanglement
\end{abstract}

\footnotetext{
${ }^{2}$ Fully documented templates are available in the elsarticle package on CTAN

* Richard Schwarzl

Email address: rschwarz@zedat.fu-berlin.de (Richard Schwarzl)
} 


\section{Introduction}

Polymer micelles with fine-tuned core-shell structures gained attention in recent years for their function as drug delivery systems in tumor therapy and topical treatment [1, 2, 3, 4, 5. They enable the transport of hydrophobic drugs in water by encapsulation in hydrophobic cores while the hydrophilic shells keep the system dispersed in water and protect the drug from the immune system [6, 7, 8, 9. In order to maximize therapeutic efficiency, the release kinetics of the drug from the nanocarriers has to be optimized and is an important factor when designing such nanocarriers [10]. Dendritic core-multishell (CMS) nanocarriers consist of a hyperbranched polar core, an inner shell and an outer shell. These nanocarriers are able to transport guest molecules in polar as well as nonpolar solvents and exhibit good tumor targeting as well as efficient topical drug delivery [11, 12, 13]. A recent study has shown that these CMS carriers can enhance penetration into the skin 12. As a transport mechanism this may allow for drugs to be released into deeper skin layers [14, 15]. In order for the drug transport via nanocarriers to be efficient, the release time of a specific drug from the carrier must be similar to the penetration time of the nanocarrier itself. Thus, reliable determination of drug-nanocarrier release kinetics is of great importance.

In this work we present a general approach to quantify the release kinetics of a drug from nano carriers and apply our method to experimental data for dexamethasone released from CMS nanocarriers. Drug release from nanocarriers is in the standard setup studied by drug diffusion through a dialysis membrane, which poses the problem of disentangling the rate of drug release from the nanocarrier and the rate of drug diffusion through the dialysis membrane 16, 17, 18, 19. We solve this problem by a two-step procedure. In the first step, we perform a calibration experiment testing the release of dexamethasone from the aqueous solution inside of a dialysis bag to an outside phosphate-buffered saline solution in the absence of nanocarriers. Here we determine the drug diffusion rate through the dialysis membrane. In the second step, we measure 
the release kinetics from nanocarriers, and use in the analysis of the data the membrane diffusion rate from the calibration experiment. For the specific dialysis membrane and CMS nanocarrier used in our experiments, we find for the membrane release rate of dexamethasone $r_{\mathrm{MI}}=0.052 \mathrm{~min}^{-1}$ and for the CMS nanocarrier release rate of dexamethasone $r_{\mathrm{NI}}=0.015 \mathrm{~min}^{-1}$. We conclude that in our specific system, the release rate from nanocarriers is only roughly three times smaller than the membrane release rate and thus both rates are of the same order. In the following section we will elaborate on the structure of the CMS nanocarriers as well as on the experimental details and the theoretical model.

\section{Materials and methods}

CMS nanocarrier preparation. The CMS nanocarriers we used in our experiments consist of a polar core surrounded by a poly ( $\varepsilon$-caprolactone) (PCL) inner shell and a monomethoxypoly(ethylene glycol) (mPEG) outer shell. The molecular weight of a single nanocarrier is $163.8 \mathrm{kDa}$. Details about synthesis and structure of the nanocarriers have been published previously [20].

Dexamethasone loading and release studies. $12 \mathrm{mg}$ of dexamethasone film was incubated with $6 \mathrm{ml}$ of CMS aqueous solution with a nanocarrier concentration of $c_{\mathrm{CMS}}=15 \mathrm{mg} / \mathrm{ml}$. After $30 \mathrm{~s}$ ultrasonication, $3 \mathrm{ml}$ of solution was withdrawn and filtered immediately with a regenerated cellulose (RC) membrane filter $(0.45 \mu \mathrm{m}$ pore size). After $3 \mathrm{~min}$ of additional ultrasonication, the remaining $3 \mathrm{ml}$ were filtered. At the start of the release experiment, $0.6 \mathrm{ml}$ of each solution was placed in a dialysis bag with a molecular weight cut-off (MWCO) of $3.5 \mathrm{kDa}$. Since dexamethasone has a molecular weight of $392.46 \mathrm{Da}$ [21], the dialysis membrane holds back the nanocarriers but lets the drug quite easily pass with a rate that we determine in the calibration experiment. The respective dialysis bag was then immersed in $30 \mathrm{ml}$ of phosphate-buffered saline (PBS). Drug release studies were performed in a bioshaker with $100 \mathrm{rpm}$ at $37^{\circ} \mathrm{C}$. Since 
the dexamthasone solution is throughly filtered before usage, we can safely assume that it corresponds to an equilibrium saturated solution and neglect the presence of aggregates in our analysis. Samples of $0.1 \mathrm{ml}$ volume were periodically removed from the outside solution and the same volume of pure PBS was added. The amount of released dexamethasone was determined by highperformance liquid chromatography (HPLC). The HPLC measurements were carried out on a Knauer Smartline-HPLC system, equipped with a reversedphase $(\mathrm{RP})-\mathrm{C} 18$ column $(250 \mathrm{~mm} \times 4 \mathrm{~mm}, 5 \mu \mathrm{m}$ particle size $)$ and an UV/VIS detector at $\lambda=245 \mathrm{~nm}$. The mobile phase was an acetonitril-water (40:60, v/v) mixture with a flow-rate of $1 \mathrm{ml} / \mathrm{min}$. For comparison, the release of free dexamethasone without CMS nanocarriers was conducted in the same condition. Each experiment was repeated three times. The release data are given by the mean of the three data sets. Errors have been calculated according to

$$
\Delta \Phi_{O}=\sqrt{\frac{1}{n-1} \sum_{j=1}^{n}\left(\Phi_{O j}-\frac{1}{n} \sum_{i=1}^{n} \Phi_{O i}\right)^{2}} .
$$

First order rate equations. We describe the release of dexamethasone using a four-state model schematically illustrated in Fig. 1. We denote the fractions of dexamethasone inside the nanocarriers, the inner solution volume, the dialysis membrane and the outer solution volume as $\Phi_{\mathrm{N}}, \Phi_{\mathrm{I}}, \Phi_{\mathrm{M}}$ and $\Phi_{\mathrm{O}}$, respectively. The release kinetics of dexamethasone from the CMS nanocarriers can be described by a system of coupled rate equations:

$$
\begin{gathered}
\frac{\mathrm{d} \Phi_{N}}{\mathrm{~d} t} \equiv \dot{\Phi}_{N}(t)=-r_{\mathrm{NI}} \Phi_{N}(t)+r_{\mathrm{IN}} \Phi_{I}(t) \\
\frac{\mathrm{d} \Phi_{I}}{\mathrm{~d} t} \equiv \dot{\Phi}_{I}(t)=-\left(r_{\mathrm{IN}}+r_{\mathrm{IM}}\right) \Phi_{I}(t)+r_{\mathrm{NI}} \Phi_{N}(t)+r_{\mathrm{MI}} \Phi_{M}(t), \\
\frac{\mathrm{d} \Phi_{M}}{\mathrm{~d} t} \equiv \dot{\Phi}_{M}(t)=-\left(r_{\mathrm{MI}}+r_{\mathrm{MO}}\right) \Phi_{M}(t)+r_{\mathrm{IM}} \Phi_{I}(t)+r_{\mathrm{OM}} \Phi_{O}(t), \\
\frac{\mathrm{d} \Phi_{O}}{\mathrm{~d} t} \equiv \dot{\Phi}_{O}(t)=-r_{\mathrm{OM}} \Phi_{O}(t)+r_{\mathrm{MO}} \Phi_{M}(t)
\end{gathered}
$$




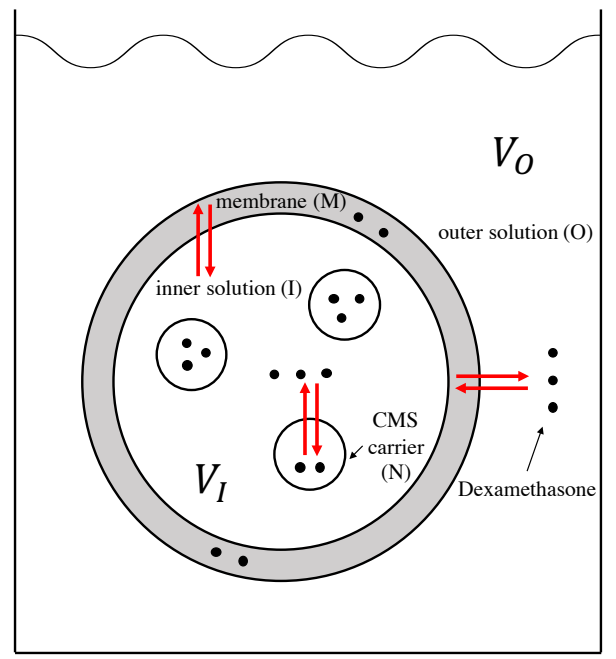

Figure 1: Schematic illustration of the experimental setup: The dialysis bag is immersed in the outer solution with a volume $V_{O}$. The black dots schematically denote dexamethasone molecules, that are either inside the nanocarriers $(\mathrm{N})$, the inner solution (I) with a volume $V_{I}$, the membrane $(\mathrm{M})$ or the outer solution $(\mathrm{O})$. During the experiment dexamethasone first diffuses from the nanoparticle interior to the inner solution, then into the membrane and finally into the outer solution. The dialysis membrane is impenetrable for the nanocarriers, leading to the desired separation of the released dexamethasone from the nanocarriers. 
where $r_{\mathrm{NI}}$ is the transition rate of going from state $N$ to state $I$ and analogous for the other rates. Two properties are used to simplify the set of equations (25). One is the conservation of the total amount of drug,

$$
\Phi_{N}(t)+\Phi_{I}(t)+\Phi_{M}(t)+\Phi_{O}(t)=1 .
$$

The other is the rate symmetry

$$
r_{\mathrm{MI}}=r_{\mathrm{MO}}
$$

which reflects that the inner and outer membrane surfaces have identical properties and areas. Note that we neglect any diffusive process inside the solutions, the nanocarriers and the membrane. This means that we assume the membrane to be relatively thin and the solutions to be well-mixed. We also neglect the finite drug loading capacity of the nanocarriers, which would lead to non-linear effects. This assumption is justified since the nanocarriers are only loaded with a few dexamethasone molecules at the beginning of the release experiments and thus should be far from their loading capacity, as we will demonstrate in the Discussion section at the end. Using eq. (6) and (7), the simplified set of equations is given by:

$$
\begin{gathered}
\dot{\Phi}_{N}(t)=-r_{\mathrm{NI}} \Phi_{N}(t)+r_{\mathrm{IN}} \Phi_{I}(t) \\
\dot{\Phi}_{I}(t)=-\left(r_{\mathrm{IM}}+r_{\mathrm{IN}}\right) \Phi_{I}(t)+r_{\mathrm{NI}} \Phi_{N}(t)+r_{\mathrm{MI}}\left(1-\Phi_{I}(t)-\Phi_{O}(t)-\Phi_{N}(t)\right) \\
\dot{\Phi}_{O}(t)=-r_{\mathrm{OM}} \Phi_{O}(t)+r_{\mathrm{MI}}\left(1-\Phi_{I}(t)-\Phi_{O}(t)-\Phi_{N}(t)\right) .
\end{gathered}
$$

In the calibration experiments in the absence of CMS nanocarriers, the above system reduces to a three-state model $(I \leftrightarrow M \leftrightarrow O)$ which is fully described by the two equations:

$$
\begin{gathered}
\dot{\Phi}_{I}(t)=-r_{\mathrm{IM}} \Phi_{I}(t)+r_{\mathrm{MI}}\left(1-\Phi_{I}(t)-\Phi_{O}(t)\right), \\
\dot{\Phi}_{O}(t)=-r_{\mathrm{OM}} \Phi_{O}(t)+r_{\mathrm{MI}}\left(1-\Phi_{I}(t)-\Phi_{O}(t)\right) .
\end{gathered}
$$


The solution of equations 11,12 for the initial condition $\Phi_{I}^{0} \equiv \Phi_{I}(0)=1$ (an explicit derivation is found in the supplement) can be given in terms of the Laplace transform of $\Phi_{O}$, denoted as $\tilde{\Phi}_{O}$ :

$$
\tilde{\Phi}_{O}(s) \equiv \int_{0}^{\infty} \Phi_{O}(t) e^{-s t} \mathrm{~d} t=\frac{r_{\mathrm{IM}} r_{\mathrm{MI}}}{s\left(s-s_{1}\right)\left(s-s_{2}\right)} .
$$

The roots $s_{1}$ and $s_{2}$ are given as:

$$
\begin{aligned}
& s_{1}=\frac{1}{2}\left(-r_{\mathrm{IM}}-2 r_{\mathrm{MI}}-r_{\mathrm{OM}}-\sqrt{r_{\mathrm{IM}}^{2}+4 r_{\mathrm{MI}}^{2}-2 r_{\mathrm{IM}} r_{\mathrm{OM}}+r_{\mathrm{OM}}^{2}}\right), \\
& s_{2}=\frac{1}{2}\left(-r_{\mathrm{IM}}-2 r_{\mathrm{MI}}-r_{\mathrm{OM}}+\sqrt{r_{\mathrm{IM}}^{2}+4 r_{\mathrm{MI}}^{2}-2 r_{\mathrm{IM}} r_{\mathrm{OM}}+r_{\mathrm{OM}}^{2}}\right) .
\end{aligned}
$$

One can further reduce the number of parameters to two by invoking the relation

$$
V_{I} r_{\mathrm{IM}}=V_{O} r_{\mathrm{OM}}
$$

which reflects that the rate for a drug molecule to hit the membrane from the inside or outside solution is inversely proportional to the solution volume, denoted by $V_{I}$ and $V_{O}$. After back transforming eq. 13 we find the time dependent fraction of dexamethasone outside the dialysis bag, $\Phi_{O}(t)$, as

$$
\Phi_{O}(t)=r_{\mathrm{IM}} r_{\mathrm{MI}} \frac{s_{1}-s_{2}+s_{2} e^{s_{1} t}-s_{1} e^{s_{2} t}}{s_{1} s_{2}\left(s_{1}-s_{2}\right)},
$$

which depends on only two parameters, $r_{\mathrm{IM}}$ and $r_{\mathrm{MI}}$, since $V_{I}=0.6 \mathrm{ml}$ and $V_{O}=30 \mathrm{ml}$ are known experimental parameters. This function is used as the model function for fitting the release data from the calibration experiments in the absence of CMS nanocarriers. The equilibrium fractions follow from the stationary solution of equations 11,12 and are denoted by an asterisk:

$$
\begin{gathered}
\frac{\Phi_{M}^{*}}{\Phi_{I}^{*}}=\frac{r_{\mathrm{IM}}}{r_{\mathrm{MI}}}, \\
\frac{\Phi_{M}^{*}}{\Phi_{O}^{*}}=\frac{r_{\mathrm{IM}}}{r_{\mathrm{MO}}} .
\end{gathered}
$$


Since the inner and the outer solution have the same dexamethasone solubility, the following relation for the equilibrium fractions holds:

$$
\frac{\Phi_{I}^{*}}{\Phi_{O}^{*}}=\frac{V_{I}}{V_{O}} .
$$

Using this assumption we can determine the equilibrium fractions as:

$$
\begin{gathered}
\Phi_{I}^{*}=\frac{r_{\mathrm{MI}} V_{I}}{r_{\mathrm{IM}} V_{I}+r_{\mathrm{MI}}\left(V_{I}+V_{O}\right)}, \\
\Phi_{O}^{*}=\frac{r_{\mathrm{MI}} V_{O}}{r_{\mathrm{IM}} V_{I}+r_{\mathrm{MI}}\left(V_{I}+V_{O}\right)}, \\
\Phi_{M}^{*}=\frac{r_{\mathrm{IM}} V_{I}}{r_{\mathrm{IM}} V_{I}+r_{\mathrm{MI}}\left(V_{I}+V_{O}\right)} .
\end{gathered}
$$

The equilibrium fraction of dexamethasone inside the dialysis membrane, $\Phi_{M}^{*}$, is called the retention fraction, and it plays an important role for the characterization of a dialysis membrane.

The solution of the rate equations in the presence of CMS nanocarriers is more involved. The derivation involves the roots of a third order polynomial, which we denote as $s_{1}, s_{2}$ and $s_{3}$ and which depend on the transition rates. A full derivation can be found in the supplement. Using that in the beginning of the release experiment, the dexamethasone is inside the inner solution $\left(\Phi_{N}^{0} \equiv \Phi_{N}(0), \Phi_{I}^{0} \equiv \Phi_{I}(0), \Phi_{N}^{0}+\Phi_{I}^{0}=1\right)$, the time dependent dexamethasone fraction in the outside solution is given as

$$
\begin{aligned}
\Phi_{O}(t)=r_{\mathrm{IM}} r_{\mathrm{MI}}\left[\frac{e^{s_{1} t}\left(r_{\mathrm{NI}}+s_{1} \Phi_{I}^{0}\right)}{s_{1}\left(s_{1}-s_{2}\right)\left(s_{1}-s_{3}\right)}+\frac{e^{s_{2} t}\left(r_{\mathrm{NI}}+s_{2} \Phi_{I}^{0}\right)}{s_{2}\left(s_{2}-s_{1}\right)\left(s_{2}-s_{3}\right)}\right. & \\
& \left.+\frac{e^{s_{3} t}\left(r_{\mathrm{NI}}+s_{3} \Phi_{I}^{0}\right)}{s_{3}\left(s_{3}-s_{1}\right)\left(s_{3}-s_{2}\right)}-\frac{r_{\mathrm{NI}}}{s_{1} s_{2} s_{3}}\right] .
\end{aligned}
$$

For the initial conditions we use the fact that the initial fractions $\Phi_{N}^{0}$ and $\Phi_{I}^{0}$ are in equilibrium,

$$
\frac{\Phi_{N}^{0}}{\Phi_{I}^{0}}=\frac{r_{\mathrm{IN}}}{r_{\mathrm{NI}}}
$$

which is in line with the fact that the experimental release data do not differ significantly for the two different loading times, $30 \mathrm{~s}$ and $3 \mathrm{~min}$ (see Fig. 3 a). 
From the maximal dexamethasone solubility in water, $c_{\mathrm{DMS}}^{\max }=0.075 \mathrm{mg} / \mathrm{ml}$, measured at room temperature, and the maximal total dexamethasone concentration in aqueous solution including CMS of a concentration $c_{\mathrm{CMS}}=15 \mathrm{mg} / \mathrm{ml}$, $c_{\mathrm{DMS}}^{\mathrm{CMS}}=0.173 \mathrm{mg} / \mathrm{ml}$, we find the initial fraction in the inner solution, $\Phi_{I}^{0}$, as

$$
\Phi_{I}^{0}=c_{\mathrm{DMS}}^{\max } / c_{\mathrm{DMS}}^{\mathrm{CMS}}=0.43
$$

and consequently the initial fraction in the nanocarriers, $\Phi_{N}^{0}$, as

$$
\Phi_{N}^{0}=1-\Phi_{I}^{0}=0.57
$$

Using the rates $r_{\mathrm{IM}}$ and $r_{\mathrm{MI}}$ that we determined from the calibration experiment, our fit function has only one remaining unknown parameter which we choose to be the rate at which dexamethasone is released from the nanocarriers to the aqueous solution volume $V_{I}$ inside the dialysis bag, $r_{N I}$.

We summarize all assumptions made in our theoretical description of the experimental release kinetics:

1. In order for the description in terms of rate equations to be valid, the drug concentration in each compartment has to be homogeneous. This means that we neglect diffusion effects inside the solutions, in the nanocarriers and in the dialysis membrane.

2. The total amount of drug in the system does not change throughout the release experiment.

3. The loading of nanocarriers in solution prior to the release experiment corresponds to thermodynamic equilibrium.

4. The membrane is thin, so that the rates $r_{\mathrm{MI}}$ and $r_{\mathrm{MO}}$ are equal.

5. The loading of the nanocarriers is far from their loading capacity so that linear kinetics is valid.

\section{Results}

Dexamethasone release kinetics through dialysis membrane: calibration experiment. We fit the average release data of the three runs of the calibration exper- 


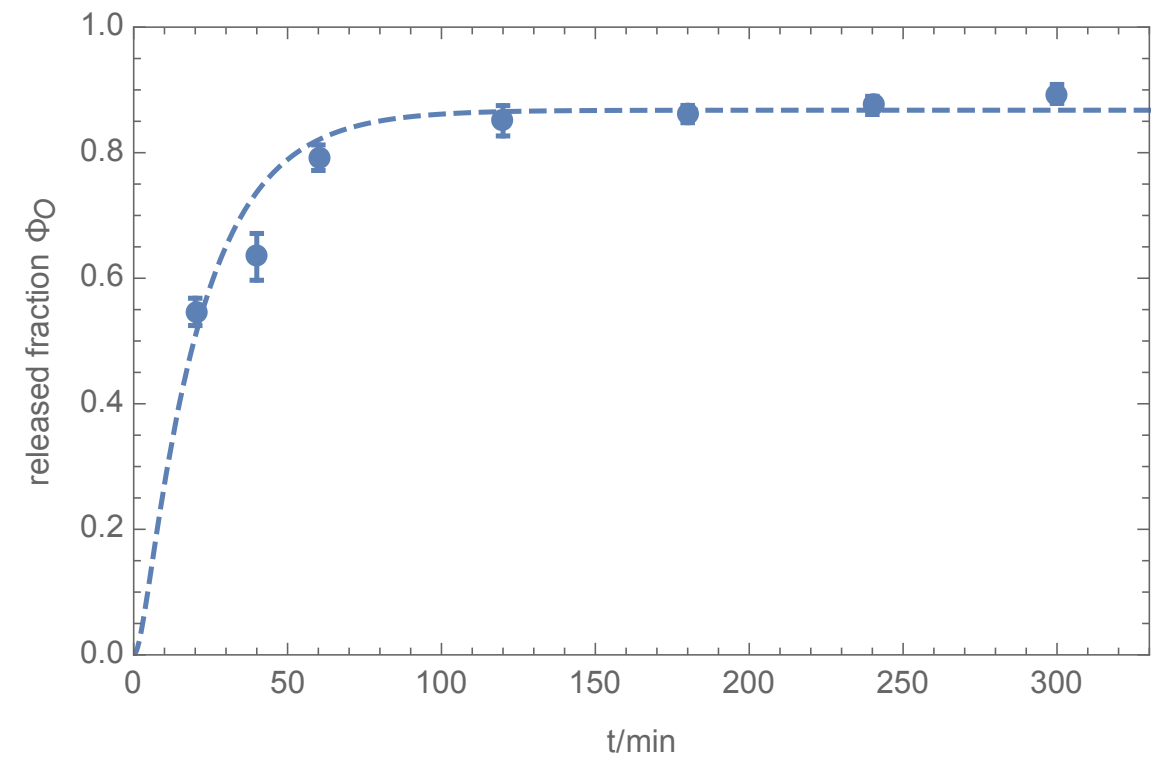

(a)

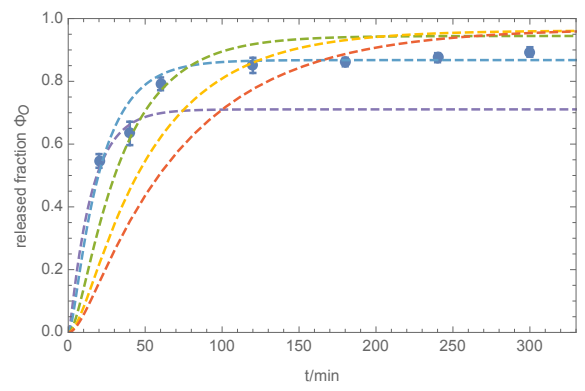

(b)

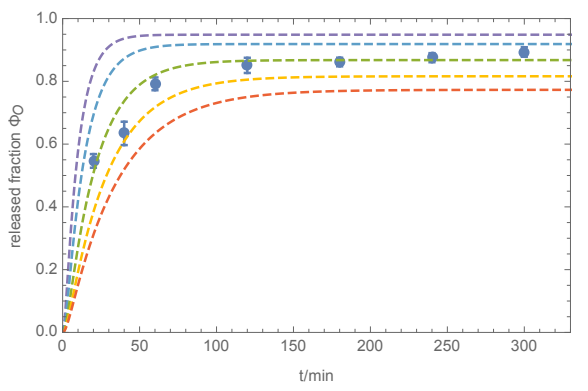

(c)

Figure 2: a) Released fraction of dexamethasone outside the dialysis bag $\Phi_{O}(t)$ from the calibration experiment (points) in absence of CMS nanocarriers and the best estimated fit according to eq. 17) (dashed line). The fit parameters are $1 / r_{\mathrm{IM}}=3 \mathrm{~min}$ and $1 / r_{\mathrm{MI}}=19 \mathrm{~min}$. b) Different curves for fixed $1 / r_{\mathrm{MI}}=19 \mathrm{~min}$ and $1 / r_{\mathrm{IM}}=1,3,10,20,30 \mathrm{~min}$ (violet, blue, green, yellow, red). c) Different curves for fixed $1 / r_{\mathrm{IM}}=3 \mathrm{~min}$ and $1 / r_{\mathrm{MI}}=5,10,19,30,40 \mathrm{~min}$ (violet, blue, green, yellow, red). 
iment in absence of CMS nanocarriers, denoted as $\Phi_{O}$, to eq. (17) using the internal function NonlinearModelFit of Mathematica (Version 10.4.0.0) 22. The squared reciprocals of the experimental errors, calculated according to eq. (1), are used as weights for the model fit. Fig. 2a displays the release data measured in the calibration experiment in the absence of CMS nanocarriers as well as a plot of the fit function in eq. (17). We find that the release rate for dexamethasone from the inner solution volume of the dialysis bag into the membrane is $r_{\mathrm{IM}}=0.342 \mathrm{~min}^{-1}\left(1 / r_{\mathrm{IM}}=3 \mathrm{~min}\right)$ with an estimated standard error of $\Delta r_{\mathrm{IM}}=0.053 \mathrm{~min}^{-1}$. The rate for dexamethasone going back from the dialysis membrane into the inner solution volume is $r_{\mathrm{MI}}=0.052 \mathrm{~min}^{-1}\left(1 / r_{\mathrm{MI}}=19 \mathrm{~min}\right)$ with an estimated standard error of $\Delta r_{\mathrm{MI}}=0.005 \mathrm{~min}^{-1}$. The remaining rates are calculated according to eq. (7) as $r_{\mathrm{MO}}=r_{\mathrm{MI}}=0.052 \mathrm{~min}^{-1}$ and eq. (16) as $r_{\mathrm{OM}}=r_{\mathrm{IM}} V_{I} / V_{O}=0.007 \mathrm{~min}^{-1}\left(1 / r_{\mathrm{OM}}=146 \mathrm{~min}\right)$. The resulting equilibrium fractions are $\Phi_{I}^{*}=r_{\mathrm{MI}} V_{I} /\left(r_{\mathrm{IM}} V_{I}+r_{\mathrm{MI}}\left(V_{I}+V_{O}\right)\right)=0.017, \Phi_{O}^{*}=\Phi_{I}^{*} V_{O} / V_{I}=$ 0.868 and the membrane retention is $\Phi_{M}^{*}=1-\Phi_{I}^{*}-\Phi_{O}^{*}=0.115$. This means that roughly $10 \%$ of dexamethasone is in equilibrium retained in the membrane, which is a significant fraction. The agreement between the data and the fit function in Fig. $2 \mathrm{a}$ is not perfect, which might point to the relevance of diffusion effects inside the dialysis membrane. Fig. $2 \mathrm{~b}$ and 2 c display curves where one of the two parameters is fixed to the best fit parameter value and the other is varied over a range of more than one order of magnitude. The curves demonstrate that the fitting procedure is quite robust.

Dexamethasone release kinetics from CMS nanocarriers. We next use the results of the fit of the calibration experiment in the absence of CMS nanocarriers for $r_{\mathrm{IM}}$ and $r_{\mathrm{MI}}$ to fit the time dependent fraction of dexamethasone $\Phi_{O}(t)$ in the presence of CMS nanocarriers inside the dialysis bag. We find the dexamethasone transition rate from the nanocarriers to the aqueous solution volume $V_{I}$, to be $r_{\mathrm{NI}}=0.015 \mathrm{~min}^{-1}\left(1 / r_{\mathrm{NI}}=66 \mathrm{~min}\right)$ with an estimated standard error of $\Delta r_{\mathrm{NI}}=0.003 \mathrm{~min}^{-1}$. Fig. 3a displays the experimental release data for two loading times $30 \mathrm{~s}$ and 3 min (yellow and green symbols) as well as release data 


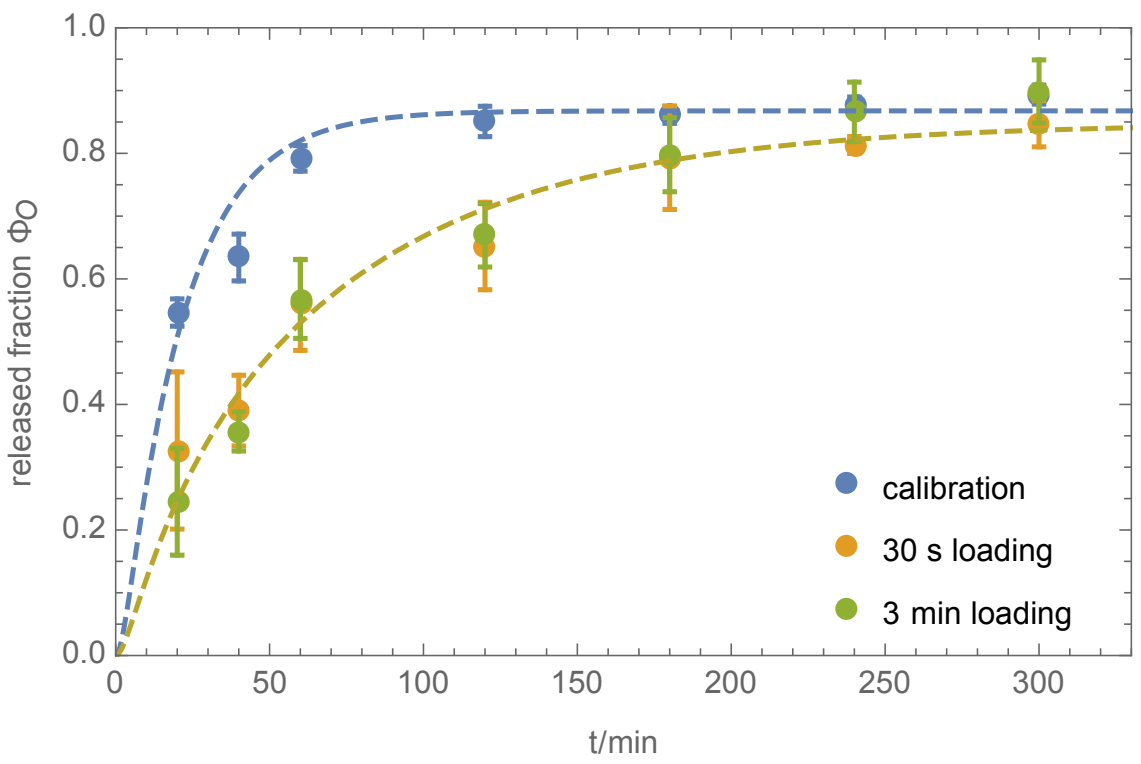

(a)

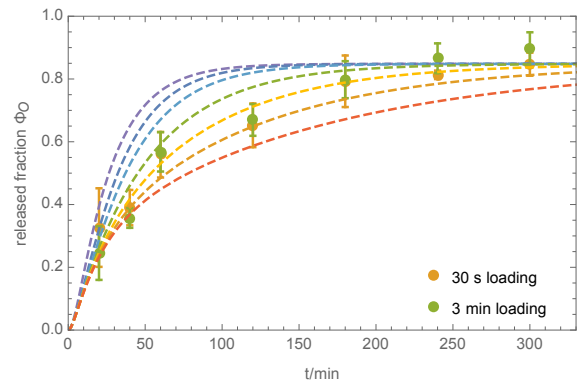

(b)

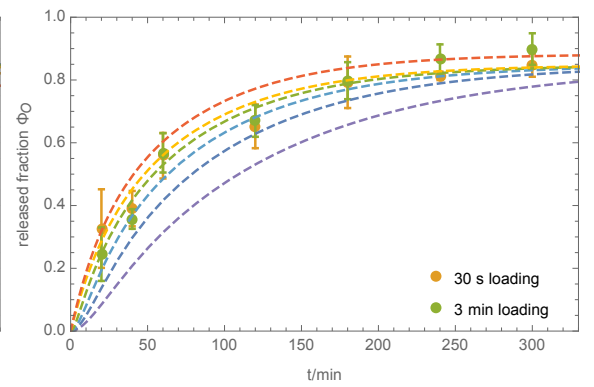

(c)

Figure 3: a) Released fraction of dexamethasone outside the dialysis bag $\Phi_{O}(t)$ from the release experiment in the absence of nanocarriers (blue points) and including nanocarriers (yellow points for $30 \mathrm{~s}$ loading time, green points for $3 \mathrm{~min}$ loading time) and the best estimated fits (dashed lines). The green curve corresponds to a dexamethasone release rate from the nanocarriers of $r_{\mathrm{NI}}=0.015 \mathrm{~min}^{-1}\left(\tau_{\mathrm{NI}}=66 \mathrm{~min}\right)$. The other two parameters, fixed by the calibration experiment, are $\tau_{\mathrm{IM}}=3 \mathrm{~min}$ and $\tau_{\mathrm{MI}}=19 \mathrm{~min}$. b) Curves for different values of $\tau_{\mathrm{NI}}=0,10,20,40,66,100,150 \mathrm{~min}$ (violet, blue, light blue, green, yellow, orange, red), $\tau_{\mathrm{IM}}=3 \mathrm{~min}$ and $\tau_{\mathrm{MI}}=19 \mathrm{~min}$. c) Curves for fixed $\tau_{\mathrm{NI}}=66 \mathrm{~min}$ and various values of $r_{\mathrm{MI}}$ and $r_{\mathrm{IM}}$ at a constant ratio, namely $\tau_{\mathrm{IM}} / \tau_{\mathrm{MI}}=0 / 0,0.03 / 0.19,3 / 19,7 / 48,15 / 97,29 / 194 \mathrm{~min} / \mathrm{min}$ (red, yellow, green, light blue, blue, violet). We see that for $\tau_{\mathrm{MI}}>\tau_{\mathrm{NI}}$ the release kinetics slows down. If on the other hand the membrane release rate $r_{\mathrm{MI}}$ becomes larger than the nanocarrier release rate $r_{\mathrm{NI}}$, the release kinetics approaches a limiting functional form. 
measured in the calibration experiment in the absence of CMS nanocarriers (blue symbols) together with the corresponding fit functions. Fig. 3b displays curves for different transition rates $r_{\mathrm{NI}}$ ranging from $150^{-1} \mathrm{~min}^{-1}$ up to the limit of $1 / r_{\mathrm{NI}} \rightarrow 0 \mathrm{~min}$. We again conclude that the fit is quite robust. In Fig. 3;, we compare the experimental data with theoretical release curves for which the membrane rates $r_{\mathrm{MI}}$ and $r_{\mathrm{IM}}$ are modified while keeping their ratio fixed. The curves demonstrate pronounced deviations from the experimental data when the membrane release rate $r_{\mathrm{MI}}$ is significantly smaller than the nanocarrier release rate $r_{\mathrm{NI}}$. In this case the drug release kinetics becomes dominated by the membrane transfer kinetics. In the opposite limit, when the membrane rate $r_{\mathrm{MI}}$ is larger than $r_{\mathrm{NI}}$, the curves approach a limiting functional form and the drug release kinetics is dominated by the release from the nano carriers. We see from this plot that for the experimental system studied by us, the kinetics of nanocarrier release and membrane transfer occur on similar time scales and thus are coupled. Clearly, for the robust analysis of dialysis release experiments it is favorable to choose a membrane that has faster release kinetics than the nanocarriers.

\section{Discussion}

By comparing experimental drug release data in the absence of nanocarriers with release data including nanocarriers, we can unambiguously estimate the release rate of dexamethasone from nanocarriers to be approximately $r_{\mathrm{NI}}=$ $0.015 \mathrm{~min}^{-1}$, which corresponds to a release time of $1 / r_{\mathrm{NI}}=66 \mathrm{~min}$. If we compare the release time from nanocarriers with the membrane release time $1 / r_{\mathrm{MO}}=19 \mathrm{~min}$, we find that they have the same order of magnitude. This means that the effect of the membrane can not be neglected when modeling the drug release kinetics from CMS nanocarriers in a typical dialysis experiment. It transpires that one has to obtain all relevant kinetic parameters in a calibration experiment in the absence of CMS nanoparticles in order to be able to fit the more complex release of dexamethasone including nanocarriers. 
We now compare our theoretical analysis of the dexamethasone release data with a much simpler kinetic model. In Fig. 4 we show the result of a fit using a single exponential decay given by

$$
\Phi_{O}(t)=1-e^{-r_{\mathrm{NI}} t}
$$

Eq. 28 can be deduced from our general model eq. 24 by taking the limits $r_{\mathrm{MO}} \rightarrow \infty$ and $r_{\mathrm{IM}} \rightarrow \infty$, corresponding to infinitely fast membrane kinetics, and using an initial condition $\Phi_{N}^{0}=1$, meaning that initially dexamethasone is entirely inside the nanocarriers. Fitting the experimental release data using this simplified model, we obtain a nanocarrier release rate of $r_{\mathrm{NI}}=0.0083 \mathrm{~min}^{-1}$ with an estimated standard error of $\Delta r_{\mathrm{NI}}=0.0012 \mathrm{~min}^{-1}$. This corresponds to a release time of $1 / r_{\mathrm{NI}}=121 \mathrm{~min}$, which is roughly two times larger than the nanocarrier release time obtained from our more realistic four-state model, which is $1 / r_{\mathrm{NI}}=66 \mathrm{~min}$. We conclude, that neglecting the effect of the membrane introduces a significant error in the resulting nanocarrier release rate. In Fig. 4 we also see that the single-exponential fit does not describe the experimental accurately over the entire time range, which we explain by the fact that the experimental data exhibit multi-exponential decay.

A subtle point of release kinetics study is the fact that typical models assume rapid mixing of the solution. In our release study we use a bioshaker in order to meet this condition. It is important to note that diffusion of the drug without shaking is not sufficiently fast to provide rapid mixing. For the sake of argument we calculate the typical diffusion time of a dexamethasone molecule within a spherical dialysis bag with a volume of $0.6 \mathrm{ml}$. The radius of the dialysis bag follows from $0.6 \cdot 10^{-6} \mathrm{~m}^{3}=\frac{4}{3} \pi r^{3}$ as $r=5.23 \mathrm{~mm}$. Using Stokes' law and the Einstein relation, we find the diffusivity of a dexamethasone molecule as $D_{\mathrm{DMS}}=k_{B} T /\left(6 \pi \eta r_{\mathrm{DMS}}\right)=\left(k_{B} 310.15 \mathrm{~K}\right) /\left(6 \pi 0.69 \cdot 10^{-3} \mathrm{~Pa} \mathrm{~s} \cdot 0.7 \mathrm{~nm}\right)=$ $0.48 \mathrm{~nm}^{2} / \mathrm{ns}$, estimating the dexamethasone radius along the long axis as $r_{\mathrm{DMS}}=$ $0.7 \mathrm{~nm}$. Consequently the mean time for a dexamethasone molecule to travel a distance of $5.23 \mathrm{~mm}$ is given by $\tau=r^{2} /\left(6 D_{\mathrm{DMS}}\right)=160 \mathrm{~min}$. Interestingly, this is more than two times larger than the release time of dexamethasone from the 


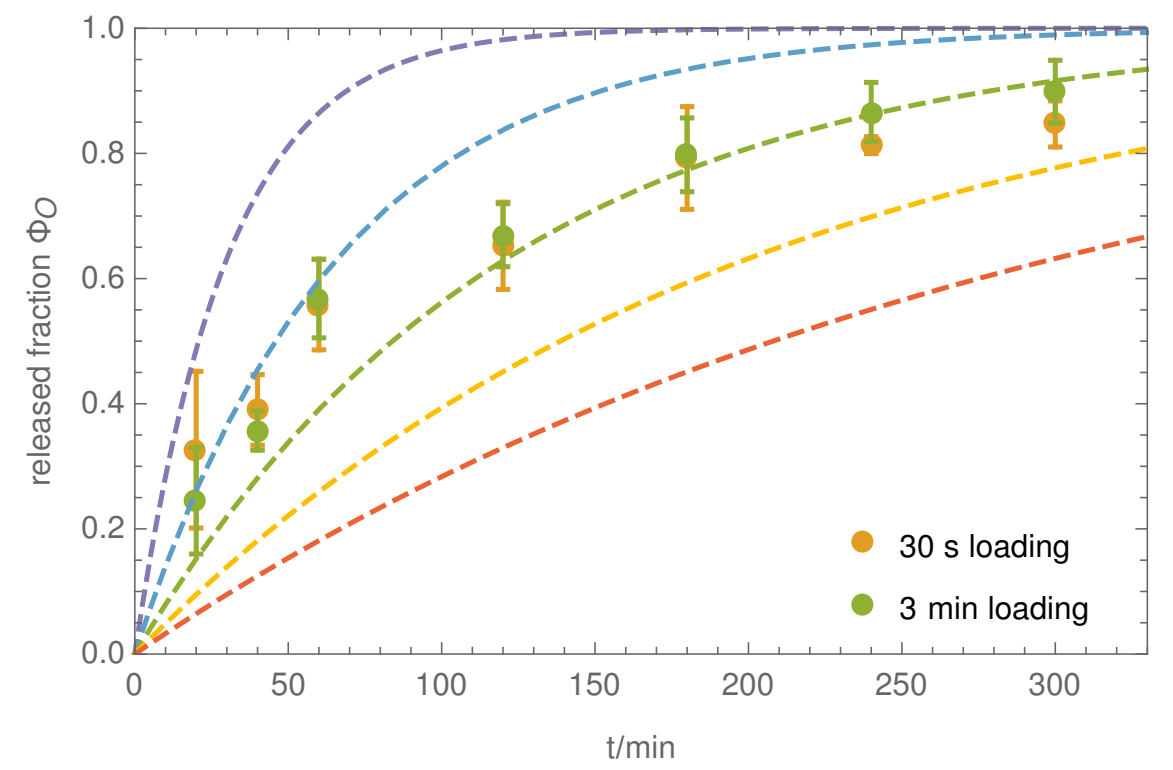

Figure 4: A single-exponential decay according to eq. 28 is compared with the experimental release data including nanocarriers. The obtained nanocarrier release rate is $r_{\mathrm{NI}}=0.0083 \mathrm{~min}^{-1}$, which is only half as large as the rate obtained from the full fourstate model $r_{\mathrm{NI}}=0.015 \mathrm{~min}^{-1}$. In addition to the best fit corresponding to $1 / r_{\mathrm{NI}}=121 \mathrm{~min}$ (green), we show curves for the different values $1 / r_{\mathrm{NI}}=30,66,200,300 \mathrm{~min}$ (violet, light blue, yellow, red). 
CMS nanocarriers $1 / r_{\mathrm{NI}}$, showing that indeed mixing of the inside of the dialysis bag is important in order to be able to model the experiment by a simple four-state model.

In order to obtain the nanocarrier loading rate per nanocarrier $\tilde{r}_{\text {IN }}$ we divide $r_{\text {IN }}$ by the nanocarrier molar concentration in the inner solution volume. The molar concentration of CMS nanocarriers $n_{\mathrm{CMS}}$ follows as $n_{\mathrm{CMS}}=c_{\mathrm{CMS}} / \mathrm{M}_{\mathrm{CMS}}=$ $(15 \mathrm{mg} / \mathrm{ml}) /(163.8 \mathrm{~kg} / \mathrm{mol})=91.6 \mathrm{mmol} / \mathrm{m}^{3}$. The transition rate coefficient $\tilde{r}_{\text {IN }}$ follows as

$$
\tilde{r}_{\mathrm{IN}}=\frac{r_{\mathrm{IN}}}{n_{\mathrm{CMS}}}=217 \frac{\mathrm{ml}}{\mathrm{min} \mathrm{mmol}} \approx 3.62 \frac{\mathrm{l}}{\mathrm{s} \mathrm{mol}} .
$$

This rescaled loading rate does not depend on the concentration of CMS nanocarriers.

Finally, we estimate the chemical potential of a dexamethasone molecule inside a CMS nanocarrier. Assuming that the CMS nanocarriers have the same density as water we estimate the total nanocarrier volume as $V_{N}=\left(c_{\mathrm{CMS}} \cdot 0.6 \mathrm{ml}\right) / c_{\mathrm{H}_{2} \mathrm{O}}=$ $(15 \mathrm{mg} / \mathrm{ml} \cdot 0.6 \mathrm{ml}) /(1000 \mathrm{mg} / \mathrm{ml})=9 \mu \mathrm{l}$. We calculate the number of CMS nanocarriers in $0.6 \mathrm{ml}$ solution with a nanocarrier concentration of $c_{\mathrm{CMS}}=$ $15 \mathrm{mg} / \mathrm{ml}$ as $N_{\mathrm{CMS}}=\left(c_{\mathrm{CMS}} \cdot 0.6 \mathrm{ml}\right) /\left(163.8 \mathrm{~kg} / \mathrm{mol} / N_{A}\right)=3.31 \cdot 10^{16}, N_{A}$ being the Avogadro constant. By dividing $V_{N}$ by the number of nanocarriers in the system, we deduce the volume of a single nanocarrier as

$$
\tilde{V}_{N}=V_{N} / N_{\mathrm{CMS}}=\frac{9 \mu \mathrm{l}}{3.31 \cdot 10^{16}}=272.0 \mathrm{~nm}^{3},
$$

corresponding to a radius $r_{\mathrm{CMS}}=4 \mathrm{~nm}$. We can calculate the number of dexamethasone molecules in $0.6 \mathrm{ml}$ of solution from the experimental concentration $c_{\mathrm{DMS}}^{\mathrm{CMS}}=0.173 \mathrm{mg} / \mathrm{ml}$ as $N_{\mathrm{DMS}}^{\mathrm{CMS}}=\left(c_{\mathrm{DMS}}^{\mathrm{CMS}} \cdot 0.6 \mathrm{ml}\right) /\left(392.46 \mathrm{~g} / \mathrm{mol} / N_{A}\right)=1.59 \cdot$ $10^{17}$. Using the fact that the concentration outside the nanocarriers corresponds to the maximal solubility $c_{\mathrm{DMS}}^{\max }$, we can define the dexamethasone concentration inside the nanocarriers $c_{\mathrm{DMS}}^{\mathrm{N}}$ via $c_{\mathrm{DMS}}^{\mathrm{CMS}} \cdot 0.6 \mathrm{ml}=c_{\mathrm{DMS}}^{\mathrm{N}} \cdot V_{N}+c_{\mathrm{DMS}}^{\max } \cdot\left(0.6 \mathrm{ml}-V_{N}\right)$, which leads to

$$
c_{\mathrm{DMS}}^{\mathrm{N}}=6.61 \mathrm{mg} / \mathrm{ml}
$$


The number of dexamethasone molecules per nanocarrier is obtained by multiplying the concentration $c_{\mathrm{DMS}}^{\mathrm{N}}$ by the volume of a single nanocarrier, $\tilde{N}_{\mathrm{DMS}}^{\mathrm{N}}=$ $c_{\mathrm{DMS}}^{\mathrm{N}} \cdot \tilde{V}_{N} /\left(392.46 \mathrm{~g} / \mathrm{mol} / N_{A}\right)=2.76$. We conclude that the number of dexamethasone molecules per nanocarrier is quite small, validating our linear theoretical description of the experimental data.

The equilibrium loading constant is obtained by dividing the dexamethasone concentration inside a nanocarrier by the concentration of dexamethasone in aqueous solution, $K=c_{\mathrm{DMS}}^{N} / c_{\mathrm{DMS}}^{\max }=(6.61 \mathrm{mg} / \mathrm{ml}) /(0.075 \mathrm{mg} / \mathrm{ml})=88$. From the equilibrium constant, we deduce the chemical potential of dexamethasone in a nanocarrier as

$$
K=e^{-\mu /\left(k_{B} T\right)} \Longrightarrow \mu=-4.48 k_{B} T \text {. }
$$

We see that dexamethasone strongly prefers the interior of a nanocarrier compared to the aqueous solution, which demonstrates that nanocarriers are highly efficient for storing and transporting hydrophobic drugs in an aqueous environment. It is interesting to compare the dexamethasone chemical potential inside a nanocarrier with the dexamethasone octanol-water partition coefficient, which is given by $\log \mathrm{P}=1.83[23$. Since $\log (K)=1.94$ is fairly close to the dexamethasone $\log \mathrm{P}$ value, we conclude that CMS nanocarriers provide a lipophilic nano-environment that is quite similar to octanol.

\section{Acknowledgement}

The authors are grateful to the SFB 1112 from the German Research Foundation (DFG) for financial support.

\section{References}

[1] S. M. Janib, A. S. Moses, J. A. MacKay, Imaging and drug delivery using theranostic nanoparticles, Advanced Drug Delivery Reviews 62 (11) (2010) 1052-1063. doi:10.1016/j.addr.2010.08.004. 
[2] I. Brigger, C. Dubernet, P. Couvreur, Nanoparticles in cancer therapy and diagnosis, Advanced Drug Delivery Reviews 64 (2012) 24-36. doi:10. 1016/j.addr.2012.09.006.

[3] K. Kazunori, K. Glenn S., Y. Masayuki, O. Teruo, S. Yasuhisa, Block copolymer micelles as vehicles for drug delivery, Journal of Controlled Release 24 (1-3) (1993) 119-132. doi:10.1016/0168-3659(93)90172-2.

[4] Z. Jin, Y. Lv, H. Cao, J. Yao, J. Zhou, W. He, L. Yin, Core-shell nanocarriers with high paclitaxel loading for passive and active targeting, Scientific Reports 6 (2016) 27559. doi:10.1038/srep27559.

[5] M. Lapteva, K. Mondon, M. Möller, R. Gurny, Y. N. Kalia, Polymeric Micelle Nanocarriers for the Cutaneous Delivery of Tacrolimus: A Targeted Approach for the Treatment of Psoriasis, Molecular Pharmaceutics 11 (9) (2014) 2989-3001. doi:10.1021/mp400639e.

[6] R. K. Kainthan, C. Mugabe, H. M. Burt, D. E. Brooks, Unimolecular Micelles Based On Hydrophobically Derivatized Hyperbranched Polyglycerols: Ligand Binding Properties, Biomacromolecules 9 (3) (2008) 886-895. doi:10.1021/bm701208p.

[7] K. Walker, J.-F. Stumbé, R. Haag, Polyester-Based, Biodegradable CoreMultishell Nanocarriers for the Transport of Hydrophobic Drugs, Polymers 8 (5) (2016) 192. doi:10.3390/polym8050192.

[8] H. M. Aliabadi, S. Elhasi, A. Mahmud, R. Gulamhusein, P. Mahdipoor, A. Lavasanifar, Encapsulation of hydrophobic drugs in polymeric micelles through co-solvent evaporation: The effect of solvent composition on micellar properties and drug loading, International Journal of Pharmaceutics 329 (1-2) (2007) 158-165. doi:10.1016/j.ijpharm.2006.08.018.

[9] J. V. Natarajan, C. Nugraha, X. W. Ng, S. Venkatraman, Sustained-release from nanocarriers: a review, Journal of Controlled Release 193 (2014) 122138. doi:10.1016/j.jconrel.2014.05.029 
[10] M. J. Johnston, S. C. Semple, S. K. Klimuk, K. Edwards, M. L. Eisenhardt, E. C. Leng, G. Karlsson, D. Yanko, P. R. Cullis, Therapeutically optimized rates of drug release can be achieved by varying the drug-to-lipid ratio in liposomal vincristine formulations, Biochimica et Biophysica Acta (BBA) Biomembranes 1758 (1) (2006) 55-64. doi:10.1016/j.bbamem.2006.01. 009 .

[11] E. Fleige, K. Achazi, K. Schaletzki, T. Triemer, R. Haag, pH-Responsive Dendritic CoreMultishell Nanocarriers, Journal of Controlled Release 185 (1) (2014) 99-108. doi:10.1016/j.jconrel.2014.04.019

[12] S. Küchler, M. R. Radowski, T. Blaschke, M. Dathe, J. Plendl, R. Haag, M. Schäfer-Korting, K. D. Kramer, Nanoparticles for skin penetration enhancement A comparison of a dendritic core-multishell-nanotransporter and solid lipid nanoparticles, European Journal of Pharmaceutics and Biopharmaceutics 71 (2) (2009) 243-250. doi:10.1016/j.ejpb.2008.08.019.

[13] D. E. Poree, M. D. Giles, L. B. Lawson, J. He, S. M. Grayson, Synthesis of Amphiphilic Star Block Copolymers and Their Evaluation as Transdermal Carriers, Biomacromolecules 12 (4) (2011) 898-906. doi: $10.1021 / \mathrm{bm} 101185 \mathrm{t}$

[14] P. Desai, R. R. Patlolla, M. Singh, Interaction of nanoparticles and cellpenetrating peptides with skin for transdermal drug delivery, Molecular Membrane Biology 27 (7) (2010) 247-259. doi:10.3109/09687688.2010. 522203

[15] P. L. Honeywell-Nguyen, J. A. Bouwstra, Vesicles as a tool for transdermal and dermal delivery, Drug Discovery Today: Technologies 2 (1) (2005) 67-74. doi:10.1016/j.ddtec.2005.05.003

[16] S. D’Souza, A Review of In Vitro Drug Release Test Methods for NanoSized Dosage Forms, Advances in Pharmaceutics 2014 (2014) 1-12. doi: $10.1155 / 2014 / 304757$ 
[17] C. Washington, Evaluation of non-sink dialysis methods for the measurement of drug release from colloids: effects of drug partition, International Journal of Pharmaceutics 56 (1) (1989) 71-74. doi:10.1016/ 0378-5173(89)90062-8.

[18] S. Modi, B. D. Anderson, Determination of Drug Release Kinetics from Nanoparticles: Overcoming Pitfalls of the Dynamic Dialysis Method, Molecular Pharmaceutics 10 (8) (2013) 3076-3089. doi:10.1021/ mp400154a.

[19] Y. Zambito, E. Pedreschi, G. Di Colo, Is dialysis a reliable method for studying drug release from nanoparticulate systems?A case study, International Journal of Pharmaceutics 434 (1-2) (2012) 28-34. doi:10.1016/j. ijpharm.2012.05.020

[20] F. Du, S. Hönzke, F. Neumann, J. Keilitz, W. Chen, N. Ma, S. Hedtrich, R. Haag, Development of biodegradable hyperbranched core-multishell nanocarriers for efficient topical drug delivery, Journal of Controlled Releasedoi:10.1016/j.jconrel.2016.06.048.

[21] S. Kim, P. A. Thiessen, E. E. Bolton, J. Chen, G. Fu, A. Gindulyte, L. Han, J. He, S. He, B. A. Shoemaker, J. Wang, B. Yu, J. Zhang, S. H. Bryant, Pubchem substance and compound databases, Nucleic Acids Research 44 (D1) (2016) D1202-D1213. doi:10.1093/nar/gkv951.

[22] Wolfram Research, Inc., Mathematica 10.4.0 (2016).

URL https://www.wolfram.com

[23] S. G. Machatha, S. H. Yalkowsky, Comparison of the octanol/water partition coefficients calculated by $\mathrm{Clog} \mathrm{P}^{\circledR}, \mathrm{ACD} \log \mathrm{P}$ and $\mathrm{KowWin}{ }^{\circledR}$ to experimentally determined values, International Journal of Pharmaceutics 294 (12) (2005) 185 - 192. doi:http://dx.doi.org/10.1016/j.ijpharm. 2005.01 .023 